\title{
ORGANIZATION OF A CIRCUIT SIMULATOR BASED ON WAVEFORM-RELAXATION METHOD
}

\author{
UMESH KUMAR* \\ EE Department, IITD Delhi, New Delhi-110016, India
}

(Received 18 J une 2001; In final form 13 August 2001)

The design of a circuit simulator based on the WR procedure is described. The relevant algorithms are presented.

Keywords: Circuit simulator; WR method; Circuit design; CAD

\section{INTRODUCTION}

Electrical circuit simulation was one of the first CAD tools developed for IC design. Circuit simulators provide precise information such as frequency response, time domain waveforms and sensitivity information.

The conventional circuit simulators like SPICE and A STA P were designed initially for the cost-effective analysis of circuits containing a few hundred transistors or less. A number of approaches have been used to improve the performances of congenital circuit simulators for the analysis of large circuits.

Recently, a relaxation method has been proposed [1-2] to provide an accurate or more accurate waveform than standard circuit simulators with up to two orders of magnitude speed improvement for large circuits. These methods attempted to exploit not only spatial scarcity but also the temporal scarcity and the unidirectional nature of MOS digital circuits. We describe the waveform relaxation simulator.

The basic idea here is to apply relaxation directly to the system of non-linear algebraic differential equations describing the circuit. As differential equations corresponding to decoupled dynamical sub circuits, each decoupled sub circuit is then analyzed for the entire simulation time interval by the standard stiffly stable integration method and the N ewton-Raphson method. The decomposition achieved allows latency to be exploited in the most natural way.

\section{ORGANIZATION OF THE WR SIMULATOR}

The WR circuit simulator will run in all interactive modes. The main routine will act as an interface between the user and processors i.e subroutines. It interprets the input commands

\footnotetext{
*E-mail: umesh@ee.iitd.ernet.in
} 
and activates the corresponding the internal processors implemented by subroutine calls. Some typical commands will be for:

(a) reading the description of the circuit from an external file

(b) continuing the execution for the W $\mathrm{R}$ iteration

(c) setting the accuracy of the analysis

(d) monitoring the waveforms at each iteration, and

(e) leaving the program.

The circuit simulator can use the Shichman-Hodges model [3] for the M OS device. All the computation will be performed in double precision and the results are also stored in double precision. As such the simulator will require a large amount of storage, especially when large circuits are analyzed e.g. for a MOS circuit containing 1000 nodes with 100 analysis time points per node. The waveform storage is required to store approximately $3 \times 1000 \times 1000$ floating point numbers (corresponding to $24 \mathrm{M} \mathrm{B}$ if each number is stored in $64 \mathrm{~B}$ its). To cope with this phenomenon a simplified storage buffering scheme can be implemented. In this scheme, the amount of primary storage allocation for the waveform is limited. When this storage is not enough to store all the waveform, a secondary storage such as a disk is used to supply the additional storage needed.

Other extensions to the WR method, which can be incorporated in the circuit simulator, involve the approximate solution of the subcircuit equation. A nother approach to simplifying the calculation is to allow the integration algorithm, which is used to solve the node waveforms of the decomposed circuits to use a larger truncation error.

\section{MATHEMATICAL FORMULATION OF WR ALGORITHM}

Two WR algorithms are proposed for analyzing an important kind of dynamical system: MOS digital integrated circuits. The Gauss Seidel (GS) and the gauss jacobi (GJ) relaxation can be used but the GS relaxation is preferred since it requires only one copy of the iterated solution as opposed to two copies required by the GJ relaxation. A lso the speed of convergence is faster, especially for MOS circuits.

Consider the first-order two-dimensional differential equations in $X(t) \in R$ on $t \in\{0$.T $\}$ :

$$
X_{1}=f_{1}\left(x_{1} \cdot x_{2} \cdot t\right) \cdot X_{1}(0)=X_{1} 0
$$

and

$$
X_{2}=f_{2}\left(x_{1} \cdot x_{2} \cdot t\right) \cdot x_{2}(0)=x_{2} 0
$$

The basic idea of the "Gauss-Seidel" waveform relaxation algorithm is to fix the waveform $X_{2}$ : [0.T]-R and solve Eq. (1a) as a one-dimensional differential equation in $X_{1}(\cdot)$. The solution thus obtained for $X_{1}$ can be substituted in the next equation which will then reduce to another first-order differential equation in one variable $X_{2}$. Equation (1b) is then resolved using the new solution for $X_{2}(t)$ and the procedure is repeated.

In this manner an iterative algorithm has been constructed. It replaces the problem of a differential equation in two variables by one of solving a sequence of differential equations in one variable. The unknown is the waveform (elements of a function space) rather than real variables.

The algorithm is a technique for time domain decoupling of differential equations. 


\section{CONCLUSION}

The WR method has proven to be an effective decomposition method for the analysis of large scale MOS circuits. It is based on the relaxation of non-linear algebraic-differential equations describing the system to be analyzed. The design and organization of a circuit simulator based on the WR method is presented. There is a lot of scope for further work with the coding of each of the modules and their linking up.

\section{References}

[1] Lelarasmee, E., Ruehii, A. E. and A ngiovanni-V incentelli, A . L. (1982). The waveform relaxation method for time domain analysis of large scale integrated circuits. IEEE Trans., CAD-1, 138-144.

[2] Newton, A. R. and Sargiovanni, A. L. (1983). Simulation. IEEE Trans., ED-30, 1184-1207.

[3] Shichman, D. and Hodges, D. A. (1968). M odelling and simulation of IGFET switching circuits. IEEE J. SolidState Circuits, SC-3, 285-289. 

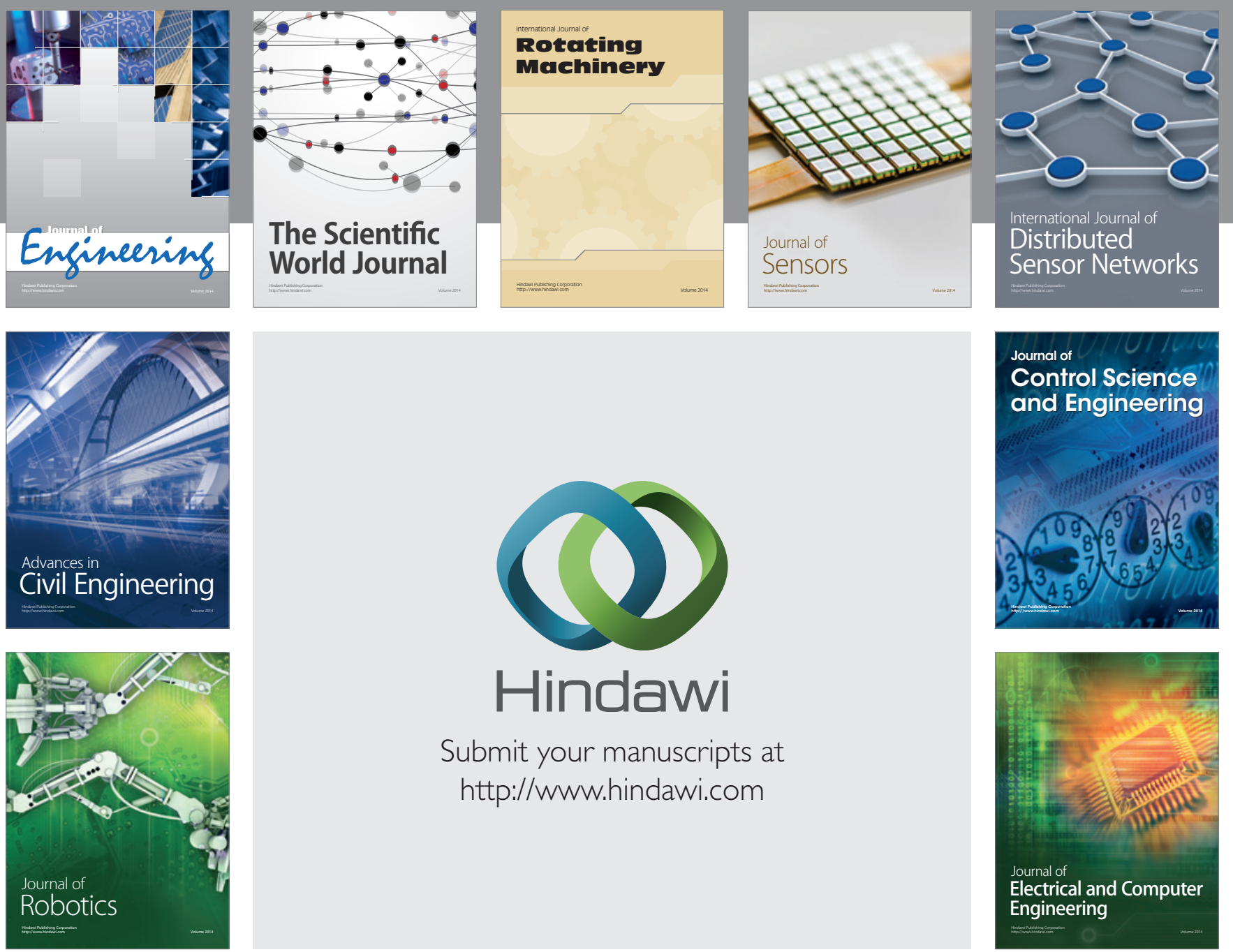

Submit your manuscripts at

http://www.hindawi.com
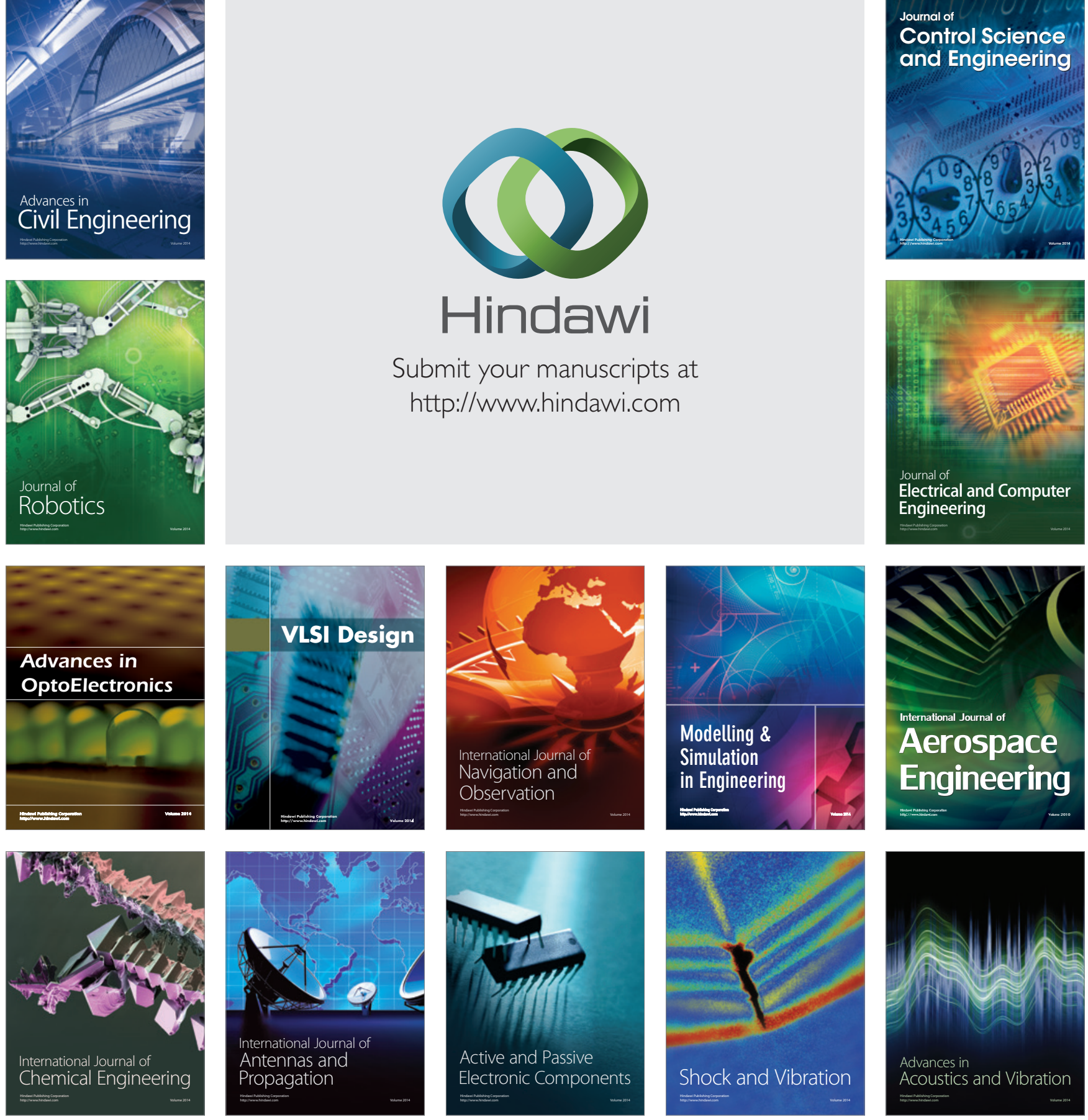